KANAZAWA 99-19 \title{
String tension and glueball masses of SU(2) QCD from perfect action for monopoles and strings *
}

\author{
S. Fujimoto, † S. Kato, M. Murata and T. Suzuki \\ Institute for Theoretical Physics, Kanazawa University, Kanazawa 920-1192, Japan
}

\begin{abstract}
We study the perfect monopole action as an infrared effective theory of SU(2) QCD. It is transformed exactly into a lattice string model. Since the monopole interactions are weak in the infrared SU(2) QCD, the string interactions become strong. The strong coupling expansion of string model shows the quantum fluctuation is small. The classical string tension is estimated analytically, and we see it is very close to the quantum one in the $\mathrm{SU}(2)$ QCD. We also discuss how to calculate the glueball mass in our model.
\end{abstract}

\section{INTRODUCTION}

The infrared effective theory of QCD is important for the analytical understanding of hadron physics. Abelian monopoles which appear after abelian projection of QCD [1] seem to be relevant dynamical degrees of freedom for infrared region [2]. Shiba and Suzuki [3] derived the monopole action from vacuum configurations obtained in Monte-Carlo simulations extending the method developed by Swendsen.

We studied the renormalized monopole action $\mathcal{S}[k]$ performing block spin transformations up to $n=8$ numerically, and saw that scaling for fixed physical length $b$ looks good [乐. If the action $\mathcal{S}[k]$ also satisfies the continuum rotational invariance, then we can regard $\mathcal{S}[k]$ as a good approximation of the renormalized trajectory(RT). In order to check this, we have to determine the correct form of physical operators on the coarse lattice.

\section{BLOCKING MONOPOLE CURRENT FROM THE CONTINUUM AND THE PERFECT OPERATOR}

Our strategy is following. (I) Let us start from the following monopole action composed of twopoint interactions between magnetic monopole currents formulated on an infinite lattice with very small lattice constant $a$ :

$S[k]=\sum_{s, s^{\prime}, \mu} k_{\mu}(s) D_{0}\left(s-s^{\prime}\right) k_{\mu}\left(s^{\prime}\right)$.

We have adopted here only parallel interactions,

* presented by S. Kato

${ }^{\dagger}$ E-mail address:kato@hep.s.kanazawa-u.ac.jp since we can avoid perpendicular interactions from short-distance terms using the current conservation. Moreover, for simplicity, we adopt only the first three Laurent expansions, i.e., Coulomb, self and nearest-neighbor interactions. Explicitly, $D_{0}\left(s-s^{\prime}\right)$ is expressed as $\beta \Delta_{L}^{-1}\left(s-s^{\prime}\right)+\alpha \delta_{s, s^{\prime}}+$ $\gamma \Delta_{L}\left(s-s^{\prime}\right)$. Here $\Delta_{L}\left(s-s^{\prime}\right)=-\sum_{\mu} \partial_{\mu} \partial_{\mu}^{\prime} \delta_{s, s^{\prime}}$ and $\partial\left(\partial^{\prime}\right)$ is the forward (backward) difference.

The monopole contribution to the potential between static abelian electric charges is derived from the following operator 5

$$
\begin{aligned}
W_{m}(\mathcal{C}) & =\exp \left(2 \pi i \sum_{s, \mu} N_{\mu}\left(s, S^{J}\right) k_{\mu}(s)\right) \\
N_{\mu}\left(s, S_{J}\right) & =\sum_{s^{\prime}} \Delta_{L}^{-1}\left(s-s^{\prime}\right) \frac{1}{2} \epsilon_{\mu \alpha \beta \gamma} \partial_{\alpha} S_{\beta \gamma}^{J}\left(s^{\prime}+\hat{\mu}\right),
\end{aligned}
$$

where $S_{\beta \gamma}^{J}\left(s^{\prime}+\hat{\mu}\right)$ is a plaquette variable satisfying $\partial_{\beta}^{\prime} S_{\beta \gamma}^{J}(s)=J_{\gamma}(s)$ and the coordinate displacement $\hat{\mu}$ is due to the interaction between dual variables.

(II) Performing a block spin transformation for monopole currents analytically, we obtain $\left\langle W_{m}(C)\right\rangle$ and the effective action on the coarse lattice $(b=n \cdot a) 8]$.

(III) Since we see the (numerically obtained) effective monopole action for SU(2) QCD in the IR region is well dominated by quadratic interactions, we regard the renormalization flow obtained in (II) as a projection of RT to the quadratic-interaction plane. We determined the couplings in (1) from the monopole action obtained by inverse Monte Carlo method. The optimal values $\kappa, m_{1}$ and $m_{2}$ for $b=2.1,2.9$ and 


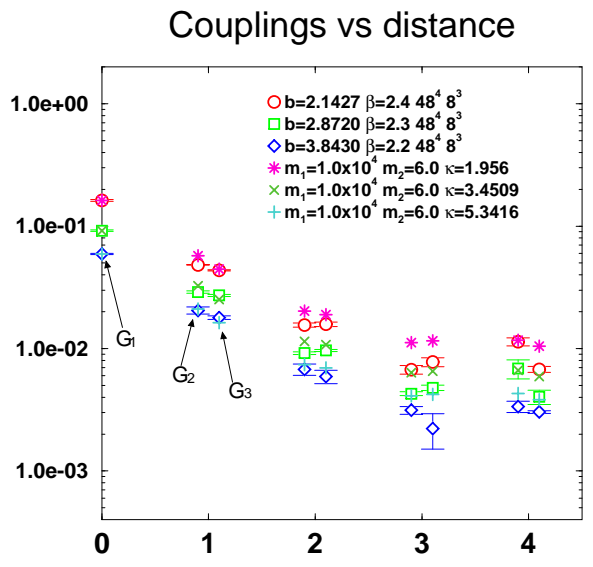

Figure 1. The $R^{2}$ dependence of the monopole action from Swendsen's method and analytical block spin transformation at $b=2.14,2.87,3.84$, where $\kappa /\left(m_{1}^{2}-m_{2}^{2}\right)=\gamma, m_{1}^{2}+m_{2}^{2}=\alpha / \gamma, m_{1}^{2} m_{2}^{2}=$ $\beta / \gamma$.

3.8 (in unit of $\sigma_{\text {phys }}^{-1 / 2}$ ) are illustrated in Figs. 1.

(IV) The above monopole action can be transformed exactly into that of the string model using BKT transformation on the lattice [9, 10]. We find finally the correct form of Wilson loop operator on the coarse lattice as follows:

$$
\begin{aligned}
& \left\langle W_{m}(\mathcal{C})\right\rangle= \\
& \exp \left\{-\pi^{2} \int_{-\infty}^{\infty} d^{4} x d^{4} y N_{\mu}(x) D_{0}^{-1}(x-y) N_{\mu}(y)\right\} \\
& \times \frac{1}{Z} \sum_{\substack{\sigma \mu \nu(s)=-\infty \\
\partial_{[\alpha} \alpha_{\mu \nu]}(s)=0}}^{\infty} \exp \{-\mathcal{S}[\sigma] \\
& \left.-2 \pi^{2} \sum_{\substack{s, s^{\prime} \\
\mu, \nu}} \sigma_{\mu \nu}(s) \partial_{\mu}^{\prime} \Delta_{L}^{-1}\left(s-s^{\prime}\right) B_{\nu}\left(s^{\prime}\right)\right\}
\end{aligned}
$$

where integer valued two-form $\sigma_{\mu \nu}(s)$ stands for string field and $\mathcal{S}[\sigma]$ is string action $[\mathbb{8}$.

\section{ROTATIONAL INVARIANCE AND THE STRING TENSION}

It turns out that the monopole action on the dual lattice is in the weak coupling region for large $b$, namely the infrared region of pure $S U(2)$ QCD.
Then the string model on the original lattice is in the strong coupling region. The strong coupling expansion on the lattice can be performed easily and quantum fluctuations terms which include more plaquettes become small[11]. Thus we consider only classical part (N-N term) in (4) below.

The plaquette variable $S_{\alpha \beta}$ in Eq.(3) for the static potential $V(b I, 0,0)$ is expressed by

$$
\begin{aligned}
S_{\alpha \beta}(z)= & \delta_{\alpha 1} \delta_{\beta 4} \delta\left(z_{2}\right) \delta\left(z_{3}\right) \theta\left(z_{1}\right) \theta\left(I b-z_{1}\right) \\
& \times \theta\left(z_{4}\right) \theta\left(T b-z_{4}\right) .
\end{aligned}
$$

To perform the integration in (4) we go to momentum space. Substituting (5) into (4), we get

$$
\begin{gathered}
\langle W(I b, 0,0, T b)\rangle \underset{\substack{T \rightarrow \infty \\
b \rightarrow \infty}}{\longrightarrow} \\
\exp \left\{-\pi^{2}\left(T I b^{2}\right) \int \frac{d^{2} p}{(2 \pi)^{2}}\left[\frac{1}{\Delta D_{0}}\right]\left(0, p_{2}, p_{3}, 0\right)\right\} .
\end{gathered}
$$

In (6), since we study large $T$ and large $b$ behaviors, we used the following expression:

$$
\lim _{T \rightarrow \infty}\left(\frac{\sin \alpha T}{\alpha}\right)^{2}=\pi T \delta(\alpha) .
$$

Similarly we can evaluate $\langle W(I b, I b, 0, T b)\rangle$. We choose the variable $S_{\alpha \beta}$ for the static potential $V(b I, I b, 0)$ as

$$
\begin{aligned}
& S_{\alpha \beta}(z)=\left(\delta_{\alpha 1} \delta_{\beta 4}+\delta_{\alpha 2} \delta_{\beta 4}\right) \delta\left(z_{3}\right) \theta\left(z_{4}\right) \theta\left(T b-z_{4}\right) \\
& \quad \times \theta\left(z_{1}\right) \theta\left(I b-z_{1}\right) \theta\left(z_{2}\right) \theta\left(I b-z_{2}\right) \delta\left(z_{1}-z_{2}\right) .
\end{aligned}
$$

The static potentials $V(I b, 0,0)$ and $V(I b, I b, 0)$ can be written as

$$
\begin{aligned}
V(I b, 0,0) & =\pi^{2}(I b) \int \frac{d^{2} p}{(2 \pi)^{2}}\left[\frac{1}{\Delta D_{0}}\right]\left(0, p_{2}, p_{3}, 0\right), \\
& =\frac{\pi \kappa I b}{2} \ln \frac{m_{1}}{m_{2}} \\
V(I b, I b, 0) & =\frac{\sqrt{2} \pi \kappa I b}{2} \ln \frac{m_{1}}{m_{2}} .
\end{aligned}
$$

The potential takes only the linear form and the continuum rotational invariance is recovered completely even for the nearest $I=1$ sites. The string tension is evaluated as $\sigma_{c l}=$ $\frac{\pi \kappa}{2} \ln \frac{m_{1}}{m_{2}}$. This is consistent with the analytical results [12. The two constants $m_{1}$ and $m_{2}$ can 
be regarded as the coherence and the penetration lengths.

The ratio $\sqrt{\sigma_{c l} / \sigma_{p h y s}}$ from the optimal values $\kappa, m_{1}$ and $m_{2}$ become 1.64, 1.56 and 1.45 for $b=2.1,2.9$ and 3.8, respectively. The scaling for physical length $b$ seems to be good, although $\sigma_{c l}$ deviate a little from physical string tension $\sigma_{\text {phys }}$.

We can also evaluate the string tension from the large flat Wilson loop not via $D_{0}$ by following expression:

$$
\begin{aligned}
& \sigma=\int_{-\pi}^{\pi} \frac{d^{2} p}{(2 \pi)^{2}} \Delta_{\mathrm{L}}^{-2}\left(k_{1}, k_{2}, 0,0\right) \\
& \times\left[\sin ^{2} \frac{k_{2}}{2} D^{-1}\left(k_{1}, k_{2}, 0,0 ; \hat{1}\right)+(1 \leftrightarrow 2)\right] .
\end{aligned}
$$

We can show this is equivalent to the above formula (9). In this case $\sqrt{\sigma_{c l} / \sigma_{\text {phys }}}$ become 1.73 , 1.59 and 1.39 for $b=2.1,2.9$ and 3.8, respectively. From these results, we see the discrepancy does not come from the systematic error of fitting $D_{0}$. It may come from the fact that we use the definition of the monopole a la DeGrand not but the real monopole in numerical study

\section{ON THE GLUEBALL MASS}

The glueball mass spectrum can be obtained by computing the correlation functions of gauge invarient local operators or Wilson loops, and extract the particle poles. For examples, one can consider two point function of operator $\mathcal{O}=$ $\operatorname{Tr}\left(F^{2}\right)$. For large $|x-y|$ it can be expanded as

$$
<\mathcal{O}(x) \mathcal{O}(y)>\simeq \sum c_{i} \exp \left(-M_{i}|x-y|\right),
$$

where $M_{i}$ are called the glueball mass.

In our model, we take the operator $\mathcal{O}$ as

$\mathcal{O}=\frac{1}{a^{4}}\left(1-W_{m}(\mathcal{C})\right)$

on the $a$-lattice. We can show this operator coinside with abelian counter part of $\mathcal{O}=\operatorname{Tr}\left(F^{2}\right)$ when lattice constant $a$ goes to zero. The evaluation of its mass is now in progress.

\section{CONCLUSIONS}

$\ddagger$ The real monopole is a monopole whose magnetic charge run from $-\infty$ to $\infty$, while the charge of the monopole a la DeGrand run from $-\left(3 n^{2}-1\right)$ to $\left(3 n^{2}-1\right)$ by definition. 13 , 14
We found the quantum perfect lattice action for monopoles and strings of hadron which describe low-energy SU(2) QCD by using analytical blockspin transformation and numerical simulation. The strong coupling expansion on the lattice for the hadronic string model works good in the infrared region of SU(2) QCD and yields the string tension. The results are almost consistent with the recent lattice results. The discrepancy may come from the definition of the monopole a la DeGrand.

\section{REFERENCES}

1. G. 't Hooft, Nucl. Phys. B190, 455 (1981).

2. T. Suzuki and I. Yotsuyanagi, Phys. Rev. D42, 4257 (1990); Nucl. Phys. B(Proc. Suppl.) 20, 236 (1991). S. Hioki et al., Phys. Lett. B272, 326 (1991) and references therein.

3. H. Shiba and T. Suzuki, Phys. Lett. B 343, 315 (1995), Phys. Lett. B 351, 519 (1995) and references therein.

4. S.Kato, S.Kitahara, N.Nakamura and T.Suzuki, Nucl. Phys. B520 (1998) 323.

5. J.D. Stack, S.D. Nieman and R.J. Wensley, Phys. Rev. D50 (1994) 3399.

6. M.N. Chernodub and M.I. Polikarpov, unpublished.

7. H. Shiba and T. Suzuki, Phys. Lett. B 333 (1994) 461.

8. S. Fujimoto et al., in this proceedings.

9. M.I.Polikarpov, U.J.Wiese and M.A.Zubkov, Phys. Lett. (1993) B309 133.

10. M.Chernodub et al, Preprint KANAZAWA 98-19, ITEP-TH-61/98.

11. S.Kato et al, Nucl.Phys.B(Proc. Suppl.)73(1999) 533.

12. T. Suzuki, Prog.Theor.Phys. 80 (1988) 929; 81 (1989) 752; S. Maedan and T. Suzuki, Prog.Theor.Phys. 80 (1988) 929; S. Maedan et al, Prog.Theor.Phys. 84 (1990) 130.

13. T.A. DeGrand and D. Toussaint, Phys. Rev. D22 (1980) 2478.

14. T.L. Ivanenko, A.V. Pochinskii and M.I. Polikarpov, Phys. Lett. B 252 (1990) 631. 